\title{
Downregulation of APRIN expression increases cancer cell proliferation via an interleukin-6/STAT3/cyclin D axis
}

\author{
MIN-SHIK SOHN ${ }^{1,2}$, MIAE KANG ${ }^{1}$, SEONG-MAN KANG ${ }^{2}$ and SANGWOO BAE ${ }^{1}$ \\ ${ }^{1}$ Division of Radiation Biomedical Research, Korea Institute of Radiological and Medical Sciences (KIRAMS), Nowon-Gu; \\ ${ }^{2}$ Graduate School of Life Sciences, Korea University, Inchonro, Seongbuk-Gu, Seoul 139-706, Republic of Korea
}

Received May 28, 2020; Accepted October 28, 2020

DOI: $10.3892 / \mathrm{ol} .2020 .12317$

\begin{abstract}
APRIN is a putative tumor suppressor whose expression is low in a variety of cancer cells. While decreased expression of APRIN leads to increased cell proliferation, unfavorable diagnosis or metastases in various cancer types, there is limited knowledge on the cellular mechanism of APRIN in cellular responses. The effect of APRIN depletion on cancer cell proliferation was examined in the present study, and the IL-6/STAT3/cyclin D axis was identified as a novel regulatory mechanism. Stable depletion of APRIN in cancer cells resulted in increased cell proliferation. Cytokine array analysis of the cells revealed that downregulation of APRIN induced secretion of interleukin-6 (IL-6) with corresponding activation of STAT3, a downstream intracellular mediator. Levels of cyclin D1 were increased in cells with APRIN depletion and cyclin D1 expression was associated with increased STAT3 binding on cyclin D1 promoter sequence; assessed by chromatin immunoprecipitation assay. The addition of an IL-6 neutralizing antibody P620 to the cell culture attenuated STAT3 activation and cyclin D1 expression in APRIN-depleted cells with corresponding decrease in cell proliferation. These experiments suggest that APRIN regulates cancer cell proliferation via an IL-6/STAT3/cyclin D axis and that targeting this axis in APRIN-associated cancer might provide a novel therapeutic approach.
\end{abstract}

\section{Introduction}

APRIN (also known as AS3 or PDS5B) is a cohesin-associated protein and is involved in the regulation of crucial cellular responses, such as chromatid cohesion, homologous recombination, DNA repair and genomic integrity $(1,2)$. APRIN-deficient mice die shortly after birth and exhibit

Correspondence to: Dr Sangwoo Bae, Division of Radiation Biomedical Research, Korea Institute of Radiological and Medical Sciences (KIRAMS), 75 Nowon-ro, Nowon-Gu, Seoul 139-706, Republic of Korea

E-mail: swbae@kcch.re.kr

Key words: APRIN (PDS5B), interleukin-6, STAT3, cyclin D1, cancer cell proliferation congenital anomalies such as heart defects, short limbs and fusion of the ribs, which underscores the essential function of the protein (3).

Moreover, APRIN has been investigated as a putative tumor suppressor. APRIN was initially studied as an androgen-induced proliferative shutoff protein that inhibits the proliferation of prostate cells that are androgen-dependent $(4,5)$. APRIN gene is located on chromosome 13 , where loss of heterozygosity is commonly detected in tumors (6). Allelic imbalance of the intragenic APRIN microsatellite repeat marker, D13S171, is associated with invasive ductal breast carcinoma (7), lung carcinoma (8), prostate cancer (9) and esophageal carcinoma (10), suggesting APRIN as a putative tumor suppressor.

While anomalies in APRIN gene expression lead to increased cell proliferation, unfavorable diagnosis, and metastases in various cancer types (6), there is limited knowledge on the cellular mechanism of APRIN in these cellular responses. Of particular note are the reports of decreased expression of APRIN in tumors (2,11-13). Low APRIN expression has been reported in tissue samples of breast tumor and is associated with high histological grade estrogen receptor-negative disease $(2,11)$. Furthermore, low expression levels of APRIN were observed in gastric and colorectal cancer, as well as in pancreatic cancer $(12,13)$.

Investigation of APRIN in cellular responses revealed distinct molecular mechanisms. The overexpression of APRIN in pancreatic cancer cells resulted in the inhibition of cell proliferation and invasion, whereas its downregulation led to enhanced proliferation and cell motility via attenuation of Ptch2 expression; suggesting that the APRIN/Ptch2 axis regulates the cellular responses of pancreatic cancer (13). APRIN associates with BRCA2 and modulates DNA damage responses as well as homologous recombination with implication in chemotherapy (2).

The present study investigated whether cancer cells might employ their unique cellular regulators to exert cellular responses upon variation in APRIN expression. The present findings demonstrate that APRIN downregulation enhances cancer cell proliferation via a novel IL-6/STAT3/cyclin D axis.

\section{Materials and methods}

Cell lines and treatments. A lung cancer cell line NCI-H460, an osteosarcoma cell line U2OS and a prostate cancer cell 
line LNCaP were obtained from American Type Culture Collection. Cell lines that stably downregulate APRIN were generated by transducing the cell lines with lentiviral particles (with $5 \times 10^{5}$ infectious units of virus) that contain either control or APRIN shRNA (Santa Cruz Biotechnology, Inc.; cat. no. SC-108080 or SC-61984-v, respectively), as specified in the instruction manual. The viral particles are provided as a ready-to-use product without the need for cell packaging processes. Control shRNA lentiviral particles encode a scrambled shRNA sequence that will not lead to the specific degradation of any known mRNA. Briefly, $5 \times 10^{4}$ cells were incubated in a 12-well plate for $24 \mathrm{~h}$ and replenished with $5 \mu \mathrm{g} / \mathrm{ml}$ polybrene-containing media. Cells were infected with $5 \times 10^{5}$ infectious units of virus. Viral particle-transduced cells were selected and maintained in puromycin-containing media. APRIN knockdown was confirmed by western blot analysis. The whole procedure to establish the stable cell lines took 30-45 days depending on the cell lines used. After lentiviral particle transduction, it took 2-3 weeks to select puromycin-resistant cells and additional 2-3 weeks to expand the antibiotic-resistant cells for experiments. The cell lines were very effective in establishing and maintaining APRIN downregulation.

NCI-H460 and LNCaP cells were cultured in RPMI-1640 media, whereas U2OS cells were grown in DMEM, supplemented with $10 \%$ fetal bovine serum (all from Welgene, Inc.), $100 \mathrm{U} / \mathrm{ml}$ penicillin and $100 \mu \mathrm{g} / \mathrm{ml}$ streptomycin. Cell cultures were incubated at $37^{\circ} \mathrm{C}$ in a humidified atmosphere of $5 \% \mathrm{CO}_{2}$.

Cell proliferation assay. Cell proliferation was measured by MTT assay, following the manufacturer's instruction (Thermo Fisher Scientific, Inc.). Absorbance was measured at $570 \mathrm{~nm}$ by using microplate reader Model 680 (Bio-Rad Laboratories, Inc.). In order to count the number of cells directly, cells were seeded on $60-\mathrm{mm}$ culture dish at a density of $2 \times 10^{4}$ cells per dish, and incubated for the indicated time period. Cells were washed with phosphate-buffered saline (PBS), and collected following trypsin treatment. Cells were counted by using Adam automated cell counter (Nano-Tek).

Cell migration assay. Cell migration assay was performed by following the manufacturer's instruction (Corning; Thermo Fisher Scientific, Inc.), with some modification. Briefly, cells were seeded on the upper layer of a 24-well Transwell plate (8.0- $\mu \mathrm{m}$ pore size; Corning; Thermo Fisher Scientific, Inc.) at a density of $1 \times 10^{4}$ cells/well with serum-free media, whereas the lower compartment was filled with RPMI-1640 culture media with $0.1 \%$ serum. After $16 \mathrm{~h}$ in the cell culture incubator, cells that migrated through the pores were visualized by staining at room temperature for $2 \mathrm{~h}$ with $0.5 \%$ crystal violet solution in $20 \%$ methanol. Stained cells were counted by microscopic observation using an INFINITY2 light optical microscope (Lumenera Corporation) at x40 magnification and recorded as migrated cell population.

Wound healing assays were conducted following the culture of cells up to $80 \%$ confluence. Cells were scratched with a pipette tip and incubated with fresh RPMI-1640 medium supplemented with $0.1 \%$ fetal bovine serum. Wound healing was observed under a light optical microscope at x10 magnification (INFINITY2; Lumenera Corporation). Wound closure was expressed as the remaining area uncovered by the cells. The scratched area at the 0 -h time-point was set to $1(n=5)$. Wound area was analyzed with captured images using the wound healing size tool of ImageJ v1.52S software (National Institutes of Health).

To carry out soft agar clonogenic assay, trypsinized cells were mixed with $1.5 \%\left(\right.$ at $55^{\circ} \mathrm{C}$ ) agar solution medium and then incubated in a $37^{\circ} \mathrm{C}$ incubator for 3-4 weeks. The number of colonies that were $>200 \mu \mathrm{m}$ in diameter were counted using a light optical microscope at x100 magnification (INFINITY2; Lumenera Corporation).

$R N A$ extraction and reverse transcription-quantitative $P C R$ $(R T-q P C R)$. Total RNA was extracted with TRIzol reagent (Invitrogen; Thermo Fisher Scientific, Inc.) by following the manufacturer's instruction. Total RNA (100 ng) was reverse-transcribed using Superscript II (Invitrogen; Thermo Fisher Scientific, Inc.), according to manufacturer's instructions. The expression of mRNA was determined in triplicate by using SYBR master mix kit (MBioTech) with a CFX96 system (Bio-Rad Laboratories, Inc.). The thermocycling conditions consisted of an initial denaturation step at $95^{\circ} \mathrm{C}$ for $10 \mathrm{~min}$, followed by 40 cycles of annealing at $60^{\circ} \mathrm{C}$ for $30 \mathrm{sec}$ and extension at $72^{\circ} \mathrm{C}$ for $15 \mathrm{sec}$. Relative mRNA expression levels were normalized to an endogenous control GAPDH expression in the corresponding samples. Relative quantification of gene expression was calculated using the $2^{-\Delta \Delta \mathrm{Cq}}$ method (14) using the CFX manager software v2.1 (Bio-Rad Laboratories, Inc.). Primers were purchased from Sigma-Aldrich (Merck KGaA). Primers used were as follows: Cyclin D1 forward, 5'-GAA CAAACAGATCATCCGCAAACA-3'; cyclin D1 reverse, 5'-TGCTCCTGGCAGGCCCGGAGGCAG-3'; IL-6 forward, 5'-GTAGCCGCCCCACACAGA-3'; IL-6 reverse, 5'-CATGTC TCCTTTCTCAGGGCTG-3'; GAPDH forward, 5'-ATGACA TCAAGAAGGTGGTG-3'; GAPDH reverse, 5'-CATACCAGG AAATGAGCTTG-3'.

Preparation of cell extracts and western blot analysis. Western blot analysis was performed as previously reported (15) with some variations in the preparation of the cell extract and the antibodies used. Cells were lysed in lysis buffer (Invitrogen), containing a protease inhibitor and phosphatase inhibitor cocktails (Sigma-Aldrich; Merck KGaA), on ice for $30 \mathrm{~min}$. The following primary antibodies (all diluted 1:1,000) were used for: APRIN (cat. no. ab70299; Abcam), STAT3 (cat. no. 9139), pSTAT3 (cat. no. 9145), cyclin D1 (cat. no. 2978) and cyclin D3 (cat. no. 2936) (Cell Signaling Technology, Inc.), cyclin A (cat. no. sc-751), cyclin B1 (cat. no. sc-594), cyclin E (cat. no. sc-248) and $\beta$-actin (cat. no. sc-81178) (Santa Cruz Biotechnology, Inc.). Peroxidase-conjugated secondary antibodies (both 1:3,000; cat. no. A90-116P for anti-mouse; cat. no. A120-101P for anti-rabbit) were purchased from Bethyl Laboratories, Inc. The experiment was repeated at least three times.

Cytokine array assay and enzyme-linked immunosorbent assay (ELISA). Control or APRIN-knockdown cells were 
seeded on $60-\mathrm{mm}$ dishes at a density of $1 \times 10^{5}$ cells/dish. After incubation for $24 \mathrm{~h}$, supernatants from the cell cultures were harvested and analyzed by using Human Cytokine Array Panel A (cat. no. ARY005B; R\&D Systems, Inc.) following the manufacturer's instructions. The levels of multiple cytokines were simultaneously detected in a sample. The levels of secreted cytokines were normalized by the cell numbers and the resulting image data was analyzed by ImageJ v1.52S program (National Institutes of Health; https://imagej.nih. gov/ij/download.html).

For ELISA assay, IL-6 ELISA kit (cat. no. D6050) was purchased from R\&D Systems, Inc. Supernatant (100 $\mu$ l) from the cell cultures was applied to the ELISA kit and processed according to manufacturer's instructions. The secreted IL-6 level was normalized by the cell numbers.

Immunofluorescence analysis. A total of $2 \times 10^{4}$ cells/well were seeded and cultured on cover slips in a 12-well plate for $24 \mathrm{~h}$. Cells were fixed with $4 \%$ paraformaldehyde for $20 \mathrm{~min}$ at room temperature, and permeabilized for $2 \mathrm{~min}$ at room temperature with $0.1 \%$ Triton X-100 solution. Specimens were blocked with $2 \%$ BSA for $1 \mathrm{~h}$ at room temperature. Immunostaining was performed with phospho-STAT3 (pSTAT3) primary antibody (1:200; cat. no. 9145; Cell Signaling Technology, Inc.) and with Alexa 488-labeled anti-rabbit IgG secondary antibody (1:500; cat. no. A-11034; Invitrogen; Thermo Fisher Scientific, Inc.). Immunofluorescence images were acquired using Axio Imager M2 microscope (Carl Zeiss AG) at x400 magnification.

Chromatin immunoprecipitation (ChIP) assay. ChIP assay was performed by using EZ-ChIP kit (cat. no. 17-371, with Taq DNA polymerase included) from EMD Millipore, following the manufacturer's instruction. Briefly, cells were treated with $1 / 10$ volume of $10 \%$ formaldehyde for $10 \mathrm{~min}$ at $37^{\circ} \mathrm{C}$ to cross-link proteins to DNA. Soluble chromatin was subjected to immunoprecipitation with anti-STAT3 antibody (Cell Signaling Technology; cat. no. 9139). Amplification of the cyclin D1 promoter sequence by PCR was carried out by using the following PCR primers: 5'-CGACCAAAGAGACAGAAC-3' and 5'-TTAACCGGGAGAAACA-3'. The PCR products were resolved on a $1.5 \%$ agarose gel and stained with ethidium bromide. Thermocycling conditions were as follows: an initial denaturation step at $94^{\circ} \mathrm{C}$ for $3 \mathrm{~min}$, followed by 32 cycles of denaturation at $94^{\circ} \mathrm{C}$ for $20 \mathrm{sec}$, annealing at $59^{\circ} \mathrm{C}$ for $30 \mathrm{sec}$ and extension at $72^{\circ} \mathrm{C}$ for $30 \mathrm{sec}$, and a final extension step at $72^{\circ} \mathrm{C}$ for $2 \mathrm{~min}$.

Statistical analysis. Data were obtained by performing three independent experiments and were presented as mean \pm SEM. Data were analyzed using GraphPad Prism 5 software (GraphPad Software, Inc.). Differences between two groups were analyzed using the Student's t-test. Multiple groups were analyzes using ANOVA followed by post hoc test, such as Bonf erroni (Figs. 1B, C, F and 4C) or Tukey's test (Fig. 4B). P<0.05 was considered to indicate a statistically significant difference.

\section{Results}

Stable downregulation of endogenous APRIN expression enhances cancer cell proliferation and migration. In order to elucidate the role of APRIN in cancer cell proliferation and migration, cancer cell lines that stably downregulate endogenous APRIN expression were established by transducing APRIN shRNA lentiviral particles. The APRIN shRNA targeted and downregulated the expression of endogenous APRIN in a human lung cancer cell line NCI-H460 and in an osteosarcoma cell line U2OS, as shown by western blot analysis (Fig. 1A). MTT cell proliferation assay revealed enhanced cell proliferation in APRIN-knockdown stable cell lines compared with the control shRNA-transduced cell lines, notably after 3 days $(\mathrm{P}<0.001$; Fig. 1B). Cell count analysis also showed similar results $(\mathrm{P}<0.05$; Fig. $1 \mathrm{C})$. These results suggest that endogenous APRIN might inhibit cancer cell proliferation.

Migration of APRIN-knockdown cell lines was investigated in Transwell migration assay. More APRIN-knockdown cells (both NCI H460 or U2OS) migrated through the membrane compared with the shRNA control cells $(\mathrm{P}=0.028$ for H460 cells; Fig. 1D). APRIN-depleted U2OS cells showed more than $50 \%$ increase in migration compared with the control $(\mathrm{P}<0.05$; Fig. 1E). The data from wound healing assay also showed similar results $(\mathrm{P}<0.001$; Fig. $1 \mathrm{~F})$. In addition, larger colonies were observed in soft agar for APRIN-depleted cells, suggesting higher malignancy upon APRIN depletion (Fig. 1G). These results suggest that endogenous APRIN might inhibit cancer cell malignancy, in terms of migration and anchorage independence.

Downregulation of endogenous APRIN increases IL-6 secretion and STAT3 activation. The mechanism underlying the effect of decreased APRIN expression on the aforementioned cancer cell responses were investigated. In order to identify such a mediator, cytokines that exhibit differential expression between control and APRIN knockdown cell lines were screened. Examination of cytokine array assay using supernatants from the culture of NCI-H460 cells revealed a prominent increase in IL-6 in APRIN-knockdown cell culture (Fig. 2A). Measurement of the intensity of the cytokine array data in Fig. 2A showed more than five-fold increase in IL-6 in the APRIN-knockdown cell sample ( $\mathrm{P}=0.0171$; Fig. 2A graph). ELISA also confirmed the data from the cytokine array; APRIN-knockdown NCI-H460 cell line secreted significantly more IL-6 into the cell culture medium compared with the control ( $\mathrm{P}=0.005$; Fig. 2B). RT-qPCR analysis of IL-6 mRNA expression showed significant increase in APRIN-knockdown cells compared with the control $(\mathrm{P}<0.0001$ for $\mathrm{H} 460 ; \mathrm{P}=0.0025$ for U2OS, and P<0.001 for LNCaP; Fig. 2C). APRIN knockdown and control cells from NCI-H460, U2OS and LNCaP background showed similar results (Fig. 2C).

Increased activation of STAT3, a downstream mediator of IL-6 receptor signaling (16), was observed in APRIN-knockdown cell lines; demonstrated by STAT3 phosphorylation at tyrosine 705 residue (pSTAT3) (Fig. 2D). Western blot analysis show that APRIN knockdown in NCI-H460, U2OS and LNCaP cells exhibited increased levels of pSTAT3 (Fig. 2D).

Immunofluorescence analysis showed increased nuclear localization of pSTAT3 in APRIN-knockdown cells (Fig. 2E). These results suggest that downregulation of APRIN expression in cancer cells might induce IL-6/STAT3-mediated 

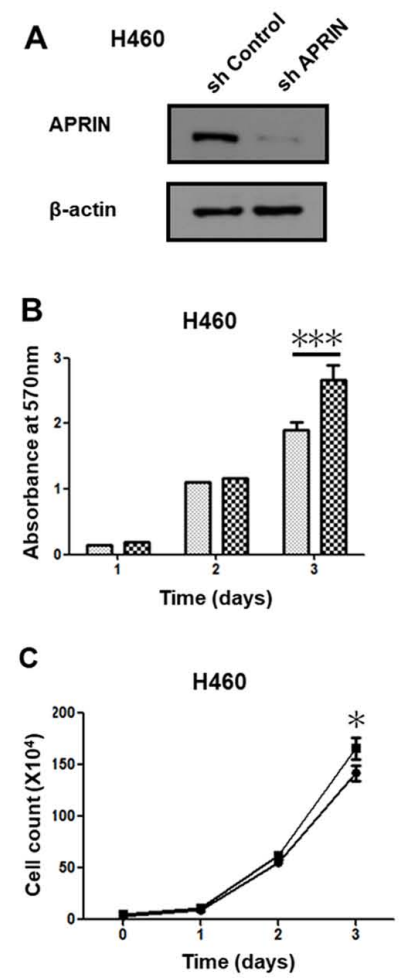

$\mathbf{F}$

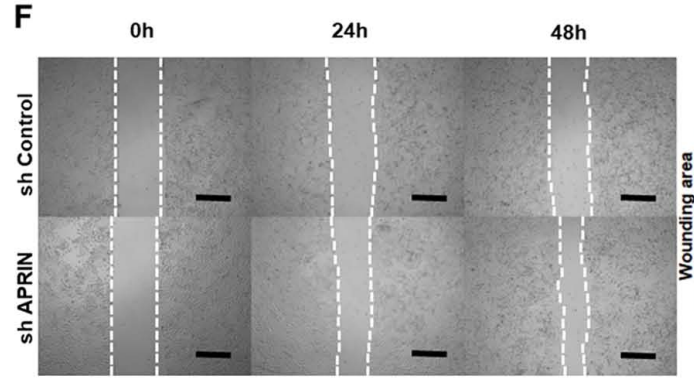

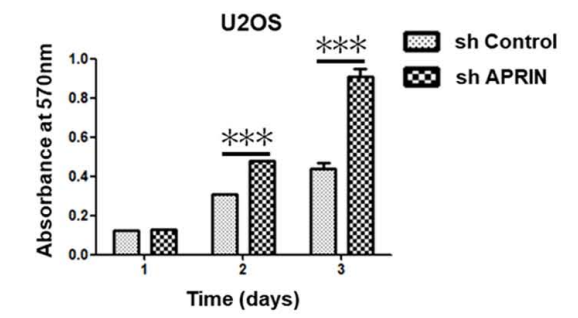
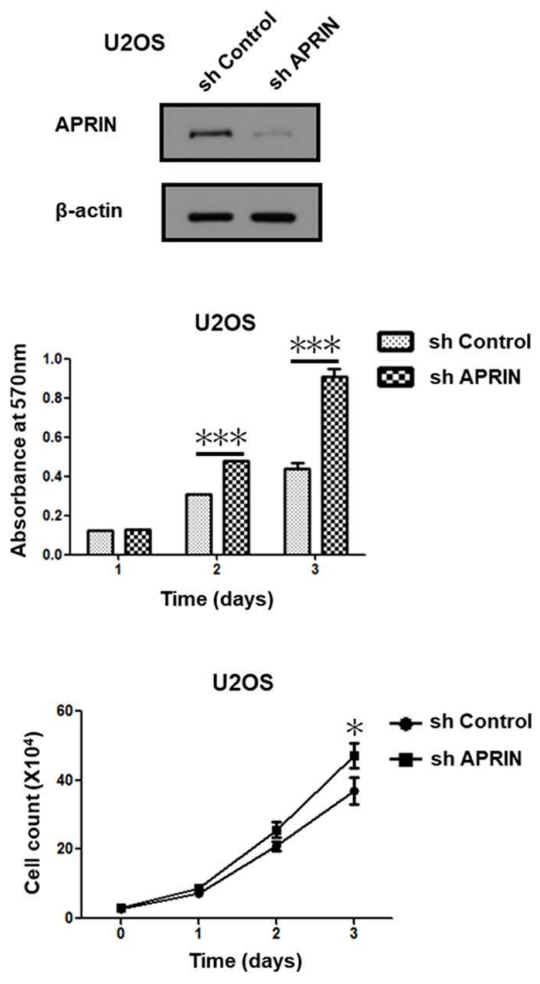

U2OS
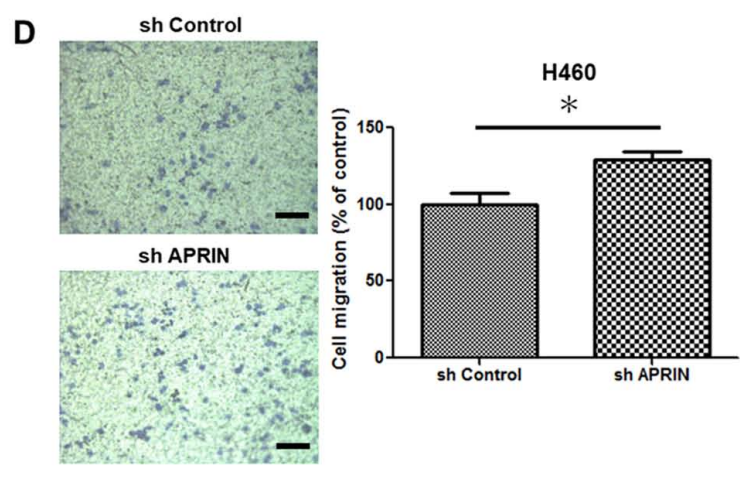

E
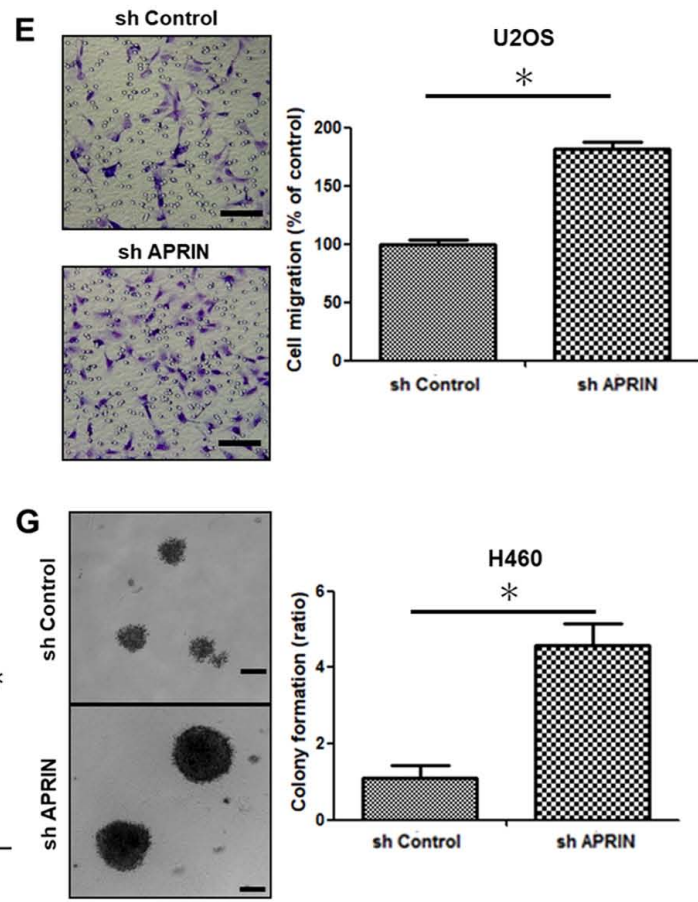

Figure 1. Downregulation of APRIN expression promotes cancer cell proliferation and migration. (A) Stable knockdown of APRIN expression by treating NCI-H460 or U2OS cells with lentiviral shRNA. APRIN expression levels were detected by western blot analysis. sh control and sh APRIN are lentiviral particles that contain either control or APRIN shRNA, respectively. (B) MTT cell proliferation assay. Proliferation of sh control or sh APRIN cells were measured. NCI-H460. (C) Cell proliferation curves of control and sh APRIN cells. (D) Images of Transwell migration assay in (D) NCI-H460 and (E) U2OS cells. Quantification of cell migration was expressed by the numbers of stained cells normalized by control. The data are presented as the mean \pm SEM $(n=3)$. Scale bar, $100 \mu \mathrm{m}$. (F) Wound healing assay of NCI-H460 cells. Increased cell migration results in decreased wounding area. Scale bar, $200 \mu \mathrm{m}$. (G) Soft agar colony-forming assay of NCI-H460 cells. Colonies of sh control and sh APRIN cells are shown. Colonies, which are $>200 \mu \mathrm{m}$ in diameter were counted and analyzed (right). ${ }^{*} \mathrm{P}<0.05 ;{ }^{* * *} \mathrm{P}<0.001$. Scale bar, $200 \mu \mathrm{m}$. sh, short hairpin RNA.

cell responses. On the other hand, endogenous APRIN may also function through modulation of IL-6/STAT3 signaling pathway.

STAT3 upregulates cyclin D expression in APRIN-knockdown cells.Inordertotest whetherthatIL-6/STAT3 signalingregulates cellular responses such as proliferation in APRIN-knockdown cells, the expression levels of cyclin family proteins were determined. Western blot analysis showed notable increase in cyclin D1 and D3 protein levels in APRIN-knockdown cells (Fig. 3A). Both NCI-H460 lung cancer cells and U2OS osteosarcoma cells showed similar results. These data suggest that the APRIN-associated cell proliferation responses might involve common regulatory components in different cell lines. In accord with the protein expression, cyclin D1 mRNA expression was also increased in APRIN knockdown cells as shown by RT-qPCR results $(\mathrm{P}<0.0001$ for $\mathrm{H} 460$ and $\mathrm{P}<0.0001$ for U2OS cells; Fig. 3B). As these results suggest a transcriptional regulation for cyclin D1 expression, whether STAT3 is involved in the regulation was examined. ChIP assay showed significantly increased association of STAT3 with cyclin D1 promoter sequence in APRIN-knockdown cells compared with control cells (Fig. 3C). These results suggest that STAT3 might be involved in the upregulation of cyclin D1 in APRIN-knockdown cells. In other words, the results suggest that endogenous APRIN might inhibit STAT3-regulated cyclin D expression.

Treatment with an IL-6-neutralizing antibody attenuates STAT3 activation, cyclin D1 mRNA expression and proliferation in APRIN-knockdown cells. In order to demonstrate that IL-6 is responsible for the downstream responses, such as increased STAT3 activation, cyclin D1 expression and proliferation in APRIN-knockdown cells, cells were treated 
A

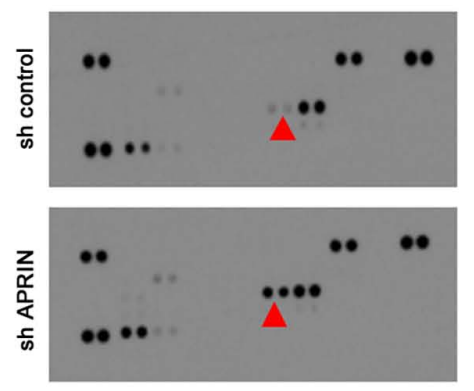

C

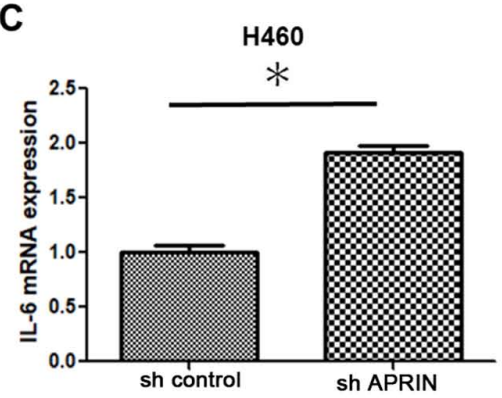

D
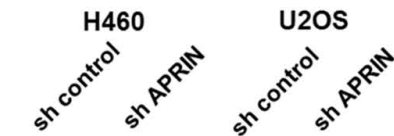

APRIN

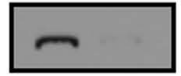

STAT3

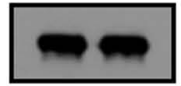

pSTAT3

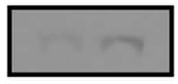

$\beta$-actin

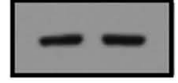

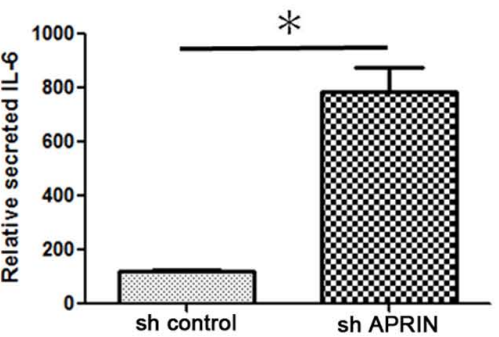

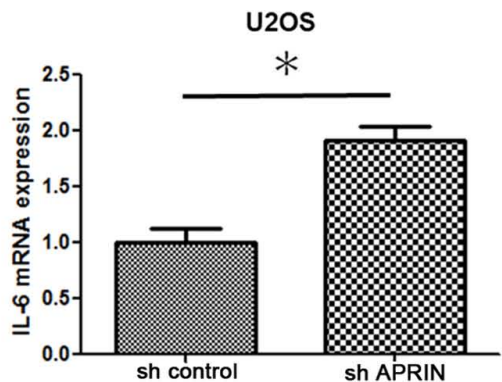

E

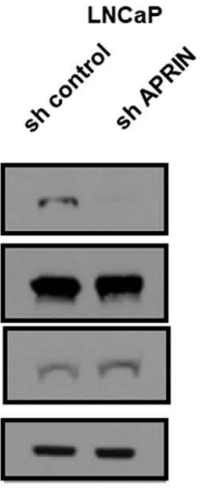

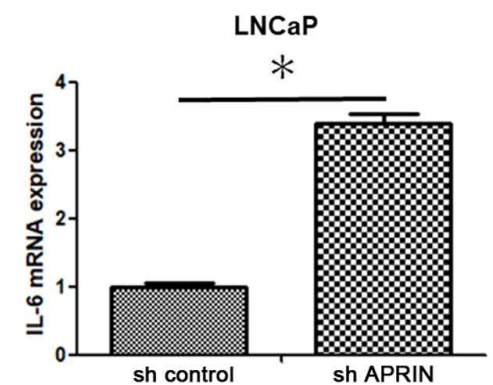

B

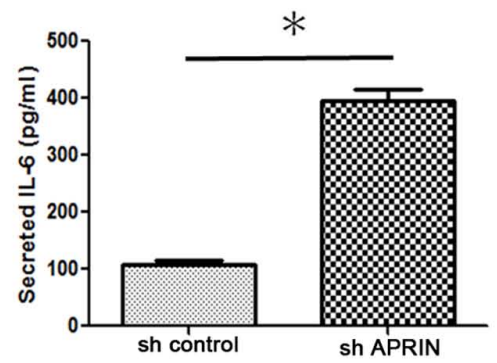

sh control
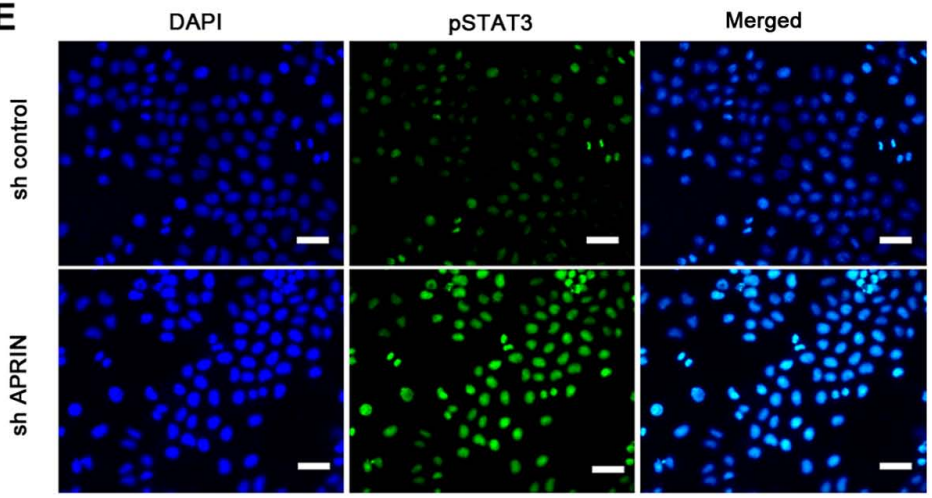

Figure 2. Induction of IL-6 secretion and elevation of activated STAT3 (pSTAT3) in APRIN-knockdown cells. (A) Cytokine array assay. Secreted IL-6 levels from control (sh control) and APRIN knockdown (sh APRIN) NCI-H460 cells were measured by using human cytokine array Panel A (left). Multiple cytokines were spotted from the cell samples. Spots of prominent differences in the levels of cytokines are pointed out by red arrow heads. Data were analyzed using ImageJ program (right graph; P=0.0171). (B) ELISA assay. Secreted IL-6 from sh control and sh APRIN NCI-H460 cells was measured by ELISA ( $\mathrm{P}=0.005$ ). (C) RT-qPCR assay of sh control and sh APRIN cells. mRNA expression of NCI-H460 ( $\mathrm{P}<0.0001)$, U2OS ( $\left.{ }^{*} \mathrm{P}=0.0025\right)$ or LNCaP ( $\left.\mathrm{P}<0.001\right)$ cells were examined. Relative expression levels of IL- 6 mRNA isolated from the cell lines were analyzed by reverse transcription-quantitative PCR. (D) Immunoblot analysis for STAT3 and pSTAT3. Cell lysates are from sh control and sh APRIN cells. (E) Immunofluorescence analysis of activated STAT3 (pSTAT3). Nuclear localization of pSTAT3 in sh control and sh APRIN NCI-H460 cells are shown. DAPI was used for nuclear staining. IL-6, interleukin-6; pSTAT; phosphorylated STAT3; sh, short hairpin RNA; ELISA, enzyme-linked immunosorbent assay.

with an IL-6-neutralizing antibody P620. Treatment of the antibody decreased phosphorylated STAT3 (pSTAT3) in APRIN-knockdown cells, as well as in control cells, whereas STAT3 protein levels in the cells were constant (Fig. 4A). Western blot analysis showed a slight increase in pSTAT3 in APRIN-knockdown cells compared with that of control cells, despite the treatment with IL-6-neutralizing antibody. This result may reflect that the amount of secreted IL-6 in APRIN-knockdown cell culture is significantly more than that of control cells and that the antibody addition resulted in partial neutralization of IL-6. These data suggest that APRIN knockdown-induced IL-6 modulates STAT3 activation.

It was also observed that the neutralizing antibody treatment significantly decreased the mRNA expression of cyclin D1 in APRIN-knockdown cells ( $\mathrm{P}<0.05$; Tukey's test; Fig. 4B).
Moreover, increased proliferation of APRIN-knockdown cells were attenuated by the antibody treatment $(\mathrm{P}<0.01$; Fig. $4 \mathrm{C})$. Therefore, APRIN may regulate cancer cell proliferation via IL-6/STAT3/cyclin D1 pathway (Fig. 4D).

\section{Discussion}

Since APRIN has been studied as a growth inhibitory gene with potential tumor suppressor functions, its association with various cancer cells has been examined, including in prostate (5), esophageal $(17,18)$, head and neck $(19)$, and pancreatic cancer cells (13). While the precise mechanism for the negative regulation of cancer cell proliferation by APRIN is still unclear, a few studies have identified distinct regulators. For example, it has been shown that overexpression of APRIN inhibits proliferation 
A

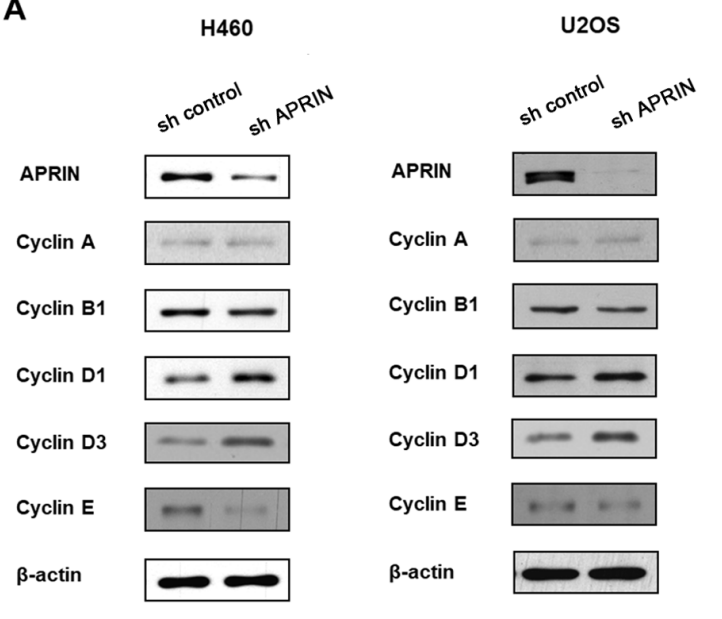

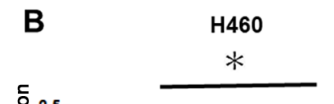
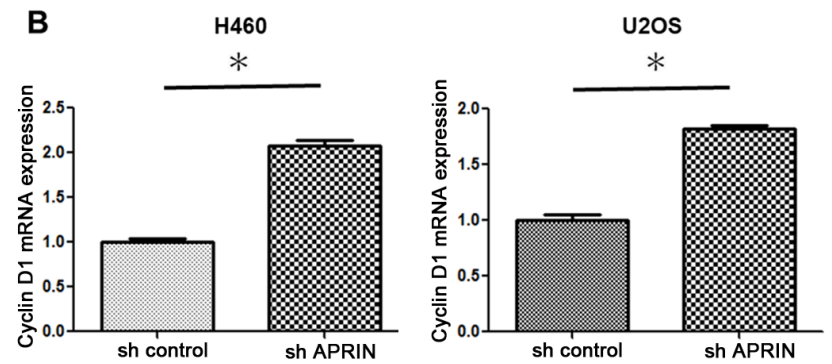

C

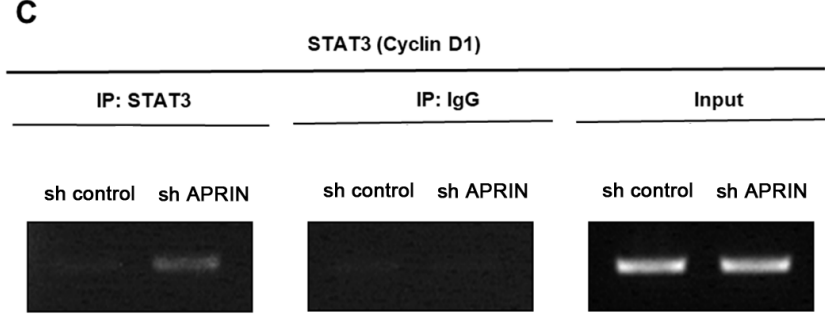

Figure 3. Enhancement of cyclin D expression by STAT3 in APRIN-knockdown cells. (A) Western blot analysis of cell cycle proteins in control (sh control) and APRIN knockdown (sh APRIN) cells. (B) Relative mRNA expression of cyclin D1 was analyzed by reverse transcription-quantitative PCR. (C) Chromatin immunoprecipitation assay to determine STAT3 association with cyclin D1 promoter. Chromatin samples were immunoprecipitated with anti-STAT3 antibody or anti-mouse IgG. Isolated DNA was amplified by PCR. Chromatin samples were prepared from sh control and sh APRIN cells. sh, short hairpin RNA. ${ }^{*} \mathrm{P}<0.0001$.

A

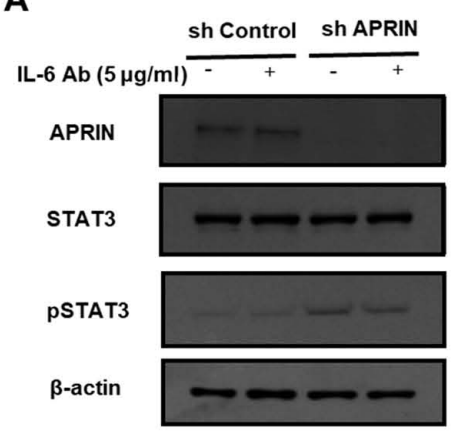

B

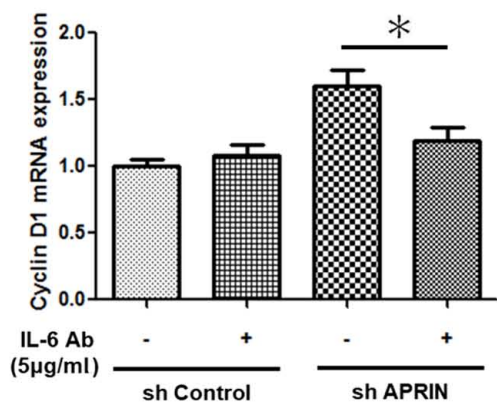

C
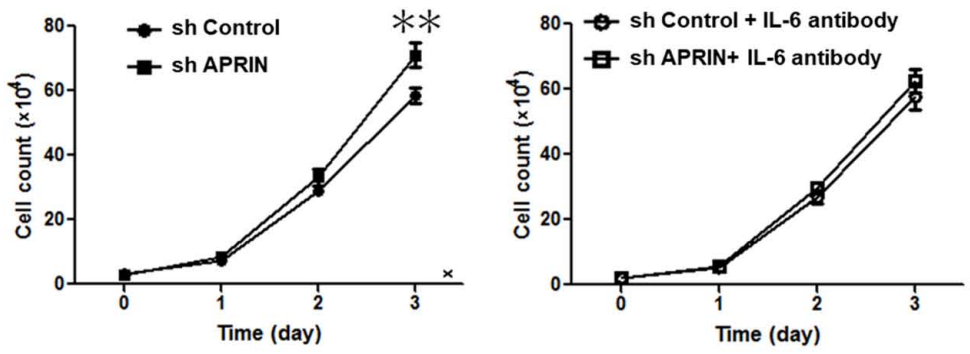

D

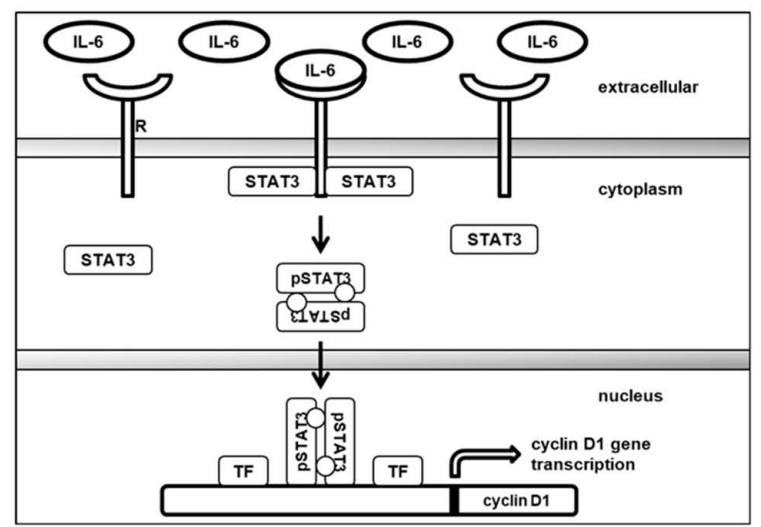

Figure 4. Treatment with an IL-6-neutralizing Ab P620 attenuates STAT3 activation and cyclin D1 expression, as well as cell proliferation. (A) Western blot analysis. Control (sh control) and APRIN knockdown (sh APRIN) NCI-H460 cells were treated with human IL-6 Ab (5 $\mu \mathrm{g} / \mathrm{ml})$ for $16 \mathrm{~h}$. Protein levels were analyzed by immunoblotting analysis. $\beta$-actin was used as a loading control. (B) Reverse transcription-quantitative PCR assay for cyclin D1 expression. sh control and sh APRIN NCI-H460 cells were treated as in (A) and cyclin D1 mRNA expression. (C) sh control and sh APRIN NCI-H460 cells were treated with P620 human IL-6 Ab $(5 \mu \mathrm{g} / \mathrm{ml})$ every $24 \mathrm{~h}$. Cell number was counted every $24 \mathrm{~h}$. Cell counts without the antibody addition (left) and with the addition of IL-6 Ab (right) are shown. (D) A schematic representation of IL-6/STAT3/cyclin D1 axis in APRIN-associated cellular responses. Increased levels of IL-6 in APRIN-depleted cells binds to its R and activates intracellular STAT3. Activated STAT3 (pSTAT3) dimerizes. The dimers are transported into the nucleus where they enhance the expression of cyclin D1 together with other TFs. "P<0.05; ** P<0.01. IL-6, interleukin-6; Ab, antibody; sh, short hairpin RNA; pSTAT3, phosphorylated STAT3; R, receptor; TFs, transcription factors.

and promotes apoptosis in P19 embryonal carcinoma cells (20). Another study suggested that APRIN upregulates Ptch2 in pancreatic cancer (PC) cells and that this APRIN/Ptch2 axis inhibits cell proliferation and invasion in PC cells (13). 
The anti-proliferative role of APRIN in cancer cells was examined in the present study. Our current findings demonstrate that APRIN downregulation enhanced cancer cell proliferation via a novel IL-6/STAT3/cyclin D axis. APRIN depletion also increased cell migration and anchorage-independent growth (Fig. 1D-G). While significant differences in the expression of typical EMT markers, such as E/N-cadherin, snail or slug (data not shown) could not be observed, wound healing assay and Transwell migration assay clearly showed enhanced cell migration in APRIN-depleted cells (Fig. 1D-F). Investigation of the unidentified factors which are responsible for the cell migration might provide insights into the APRIN-associated cellular responses. Notably, it would be interesting to screen IL-6/STAT3-regulated factors that are involved in cell migration.

Stable downregulation of APRIN expression in a lung cancer cell line NCI-H460 resulted in prominent increase in IL-6 (Fig. 2). Since the cytokine levels were measured by using culture media supernatant, these findings demonstrated that IL-6 is secreted from the cell line and is responsible for the downstream cellular responses. Indeed, one of the downstream regulators, STAT3 was activated in the APRIN-downregulated cell line (Fig. 2D). Treatment of the cell culture with IL-6-neutralizing antibody attenuated STAT3 activation and its downstream target gene cyclin D1 expression (Fig. 4). These findings demonstrate that prominent paracrine production of IL-6 is responsible for the enhanced activation of STAT3 in APRIN-downregulated cells.

It was reported that many lung cancer cell lines exhibit variable levels of activated STAT3 (pSTAT3) (21). Identification of the regulatory factors that are responsible for STAT3 activation might have implications for the development of targeted therapy in cancer (22). Depletion of STAT3 itself in the cells affected cell viability limiting further investigation. However, the present results showed that blockade of IL-6 secretion attenuates the enhanced growth of the APRIN-depleted lung cancer cell line (Fig. 4C), demonstrating potential therapeutic benefit of IL-6 inhibition.

While IL-6 is upregulated in lung cancer patient and is associated with decreased cancer survival (23-25), its association with APRIN is first shown in the present study. The mechanism by which APRIN downregulation leads to IL-6 upregulation in lung cancer cells is unknown. APRIN depletion might cause pleiotropic effects in cellular responses including activities of transcription factors. Transcriptional mediators of IL- 6 gene such as NF- $\kappa \mathrm{B}, \mathrm{AP}-1$ and CREB were reported (26-28). Interestingly, the involvement of STAT3 with NF- $\kappa \mathrm{B}$ has been suggested in IL-6 gene induction (29). Thus, sequence of events may be envisioned where APRIN depletion activates a multitude of transcription factors and some of these in turn induce IL-6 expression. Increased IL-6 may activate downstream transcription factors including STAT3, which might reciprocally amplify IL-6 production. The resulting augmented activity of IL-6 and STAT3 might lead to enhanced expression of cyclin D1 (Fig. 4D). Further examination should reveal how APRIN depletion regulates the IL-6/STAT3/cyclin D axis.

A study suggests that loss of APRIN expression could sensitize breast cancer cells to DNA damaging agents (2). Characterizing the responses of various cancer cells with aberrant APRIN expression to diverse therapeutic agents, may provide crucial data to develop therapeutic approaches for APRIN/IL-6/STAT3-associated cancer.

\section{Acknowledgements}

Not applicable.

\section{Funding}

This study was supported by a grant of the Korea Institute of Radiological and Medical Sciences (grant no. 50531-2020) funded by Ministry of Science and ICT, Republic of Korea.

\section{Availability of data and materials}

All data generated or analyzed during this study are included in this published article.

\section{Authors' contributions}

SB and SK designed and supervised the experiments. MS and MK conducted the experiments. SB, SK and MS wrote the manuscript. All authors read and approved the final manuscript.

\section{Ethics approval and consent to participate}

Not applicable.

\section{Patient consent for publication}

Not applicable.

\section{Competing interests}

The authors declare that they have no competing interests.

\section{References}

1. Xiong B and Gerton JL: Regulators of the cohesin network. Annu Rev Biochem 79: 131-153, 2010.

2. Brough R, Bajrami I, Vatcheva R, Natrajan R, Reis-Filho JS, Lord CJ and Ashworth A: APRIN is a cell cycle specific BRCA2-interacting protein required for genome integrity and a predictor of outcome after chemotherapy in breast cancer. EMBO J 31: 1160-1176, 2012

3. Zhang B, Jain S, Song H, Fu M, Heuckeroth RO, Erlich JM, Jay PY and Milbrandt J: Mice lacking sister chromatid cohesion protein PDS5B exhibit developmental abnormalities reminiscent of Cornelia de Lange syndrome. Development 134: 3191-3201, 2007.

4. Geck P, Szelei J, Jimenez J,Lin TM, Sonnenschein C and Soto AM: Expression of novel genes linked to the androgen-induced, proliferative shutoff in prostate cancer cells. J Steroid Biochem Mol Biol 63: 211-218, 1997.

5. Geck P, Maffini MV, Szelei J, Sonnenschein C and Soto AM: Androgen-induced proliferative quiescence in prostate cancer cells: The role of AS3 as its mediator. Proc Natl Acad Sci USA 97: 10185-10190, 2000.

6. Geck P, Sonnenschein C and Soto AM: The D13S171 marker, misannotated to BRCA2, links the AS3 gene to various cancers. Am J Hum Genet 69: 461-463, 2001.

7. Beckmann MW, Picard F, An HX, van Roeyen CR, Dominik SI, Mosny DS, Schnürch HG, Bender HG and Niederacher D: Clinical impact of detection of loss of heterozygosity of BRCA1 and BRCA2 markers in sporadic breast cancer. Br J Cancer 73: 1220-1226, 1996. 
8. Gorgoulis VG, Kotsinas A, Zacharatos P, Mariatos G, Liloglou T, Tsoli E, Kokotas S, Fassoulas C, Field JK and Kittas C: Association of allelic imbalance at locus D13S171 (BRCA2) and 553 alterations with tumor kinetics and chromosomal instability (aneuploidy) in nonsmall cell lung carcinoma. Cancer 89 : 1933-1945, 2000.

9. Edwards SM, Dunsmuir WD, Gillett CE, Lakhani SR, Corbishley C, Young M, Kirby RS, Dearnaley DP, Dowe A, Ardern-Jones A, et al; CRC/BPG UK Familial Prostate Cancer Study Collaborators: Immunohistochemical expression of BRCA2 protein and allelic loss at the BRCA2 locus in prostate cancer. Int J Cancer 78: 1-7, 1998.

10. Harada H, Tanaka H, Shimada Y, Shinoda M, Imamura M and Ishizaki K: Lymph node metastasis is associated with allelic loss on chromosome 13q12-13 in esophageal squamous cell carcinoma. Cancer Res 59: 3724-3729, 1999.

11. Chen W, Salto-Tellez M, Palanisamy N, Ganesan K, Hou Q, Tan LK, Sii LH, Ito K, Tan B, Wu J, et al: Targets of genome copy number reduction in primary breast cancers identified by integrative genomics. Genes Chromosomes Cancer 46: 288-301, 2007.

12. Kim MS, An CH, Yoo NJ and Lee SH: Frameshift mutations of chromosome cohesion-related genes SGOL1 and PDS5B in gastric and colorectal cancers with high microsatellite instability. Hum Pathol 44: 2234-2240, 2013.

13. Ma J, Cui Y, Cao T, Xu H, Shi Y, Xia J, Tao Y and Wang ZP: PDS5B regulates cell proliferation and motility via upregulation of Ptch2 in pancreatic cancer cells. Cancer Lett 460: 65-74, 2019.

14. Livak KJ and Schmittgen TD: Analysis of relative gene expression data using real-time quantitative PCR and the 2(-Delta DeltaC(T)) method. Methods 25: 402-408, 2001.

15. Chung HJ, Yoon SI, Shin SH, Koh YA, Lee SJ, Lee YS and Bae S: p53-mediated enhancement of radiosensitivity by selenophosphate synthetase 1 overexpression. J Cell Physiol 209: 131-141, 2006.

16. Levy DE and Lee CK: What does Stat3 do? J Clin Invest 109: $1143-1148,2002$.

17. Harada H, Uchida N, Shimada Y, Kumimoto H, Shinoda M, Imamura $\mathrm{M}$ and Ishizaki K: Polymorphism and allelic loss at the AS3 locus on 13q12-13 in esophageal squamous cell carcinoma Int J Oncol 18: 1003-1007, 2001

18. Zhang Y, Huang X, Qi J, Yan C, Xu X, Han Y and Wang M: Correlation of genomic and expression alterations of AS3 with esophageal squamous cell carcinoma. J Genet Genomics 35: 267-271,2008

19. Reis EM, Ojopi EP, Alberto FL, Rahal P, Tsukumo F, Mancini UM, Guimarães GS, Thompson GM, Camacho C, Miracca E, et al; Head and Neck Annotation Consortium: Large-scale transcriptome analyses reveal new genetic marker candidates of head, neck, and thyroid cancer. Cancer Res 65 : $1693-1699,2005$.
20. Zhou X, Kong X, Xu W and Chen J: Overexpression of APRIN inhibits differentiation and proliferation and promotes apoptosis in P19 embryonal carcinoma cells. Mol Biol Rep 40: 491-495, 2013.

21. Gao SP, Mark KG, Leslie K, Pao W, Motoi N, Gerald WL, Travis WD, Bornmann W, Veach D, Clarkson B, et al: Mutations in the EGFR kinase domain mediate STAT3 activation via IL-6 production in human lung adenocarcinomas. J Clin Invest 117: 3846-3856, 2007

22. $\mathrm{Yu} \mathrm{H}$ and Jove R: The STATs of cancer--new molecular targets come of age. Nat Rev Cancer 4: 97-105, 2004.

23. Chang CH, Hsiao CF, Yeh YM, Chang GC, Tsai YH, Chen YM, Huang MS, Chen HL, Li YJ, Yang PC, et al: Circulating interleukin-6 level is a prognostic marker for survival in advanced nonsmall cell lung cancer patients treated with chemotherapy. Int J Cancer 132: 1977-1985, 2013.

24. Pine SR, Mechanic LE, Enewold L, Chaturvedi AK, Katki HA, Zheng YL, Bowman ED, Engels EA, Caporaso NE and Harris CC: Increased levels of circulating interleukin 6, interleukin 8, C-reactive protein, and risk of lung cancer. J Natl Cancer Inst 103: 1112-1122, 2011.

25. Songür N, Kuru B, Kalkan F, Özdilekcan C, Cakmak H and Hizel N: Serum interleukin-6 levels correlate with malnutrition and survival in patients with advanced non-small cell lung cancer. Tumori 90: 196-200, 2004.

26. Xiao W, Hodge DR, Wang L, Yang X, Zhang X and Farrar WL: Co-operative functions between nuclear factors NFkappaB and CCAT/enhancer-binding protein- $\beta$ (C/EBP- $\beta$ ) regulate the IL-6 promoter in autocrine human prostate cancer cells. Prostate 61: 354-370, 2004.

27. Sehgal PB: Regulation of IL6 gene expression. Res Immunol 143 724-734, 1992.

28. Squarize CH, Castilho RM, Sriuranpong V, Pinto DS Jr and Gutkind JS: Molecular cross-talk between the NFkappaB and STAT3 signaling pathways in head and neck squamous cell carcinoma. Neoplasia 8: 733-746, 2006.

29. Yang J, Liao X, Agarwal MK, Barnes L, Auron PE and Stark GR: Unphosphorylated STAT3 accumulates in response to IL-6 and activates transcription by binding to NFkappaB. Genes Dev 21: 1396-1408, 2007.

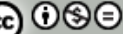

This work is licensed under a Creative Commons Attribution-NonCommercial-NoDerivatives 4.0 International (CC BY-NC-ND 4.0) License. 